\title{
BMJ Open Analysis of sensorimotor control in people with and without neck pain using inertial sensor technology: study protocol for a 1-year longitudinal prospective observational study
}

\author{
Filippo Moggioli (D) , ${ }^{1}$ Tomas Pérez-Fernández (D) , ${ }^{1}$ Sonia Liébana, ${ }^{1}$ \\ Elena Bocos Corredor, ${ }^{1}$ Susan Armijo-Olivo, ${ }^{2,3}$ Josue Fernandez-Carnero, ,,5,6 \\ Rafael Raya, ${ }^{7,8}$ Pablo Conde, ${ }^{1}$ Oscar Rodríguez-López, ${ }^{1}$ Cristina Sánchez, ${ }^{7}$ \\ Aitor Martín-Pintado-Zugasti (i) ${ }^{1}$
}

To cite: Moggioli F, PérezFernández T, Liébana S, et al. Analysis of sensorimotor control in people with and without neck pain using inertial sensor technology: study protocol for a 1-year longitudinal prospective observational study. BMJ Open 2022;12:e58190. doi:10.1136/ bmjopen-2021-058190

- Prepublication history and additional supplemental material for this paper are available online. To view these files, please visit the journal online (http://dx.doi.org/10.1136/ bmjopen-2021-058190).

Received 13 October 2021 Accepted 14 January 2022

Check for updates

(c) Author(s) (or their employer(s)) 2022. Re-use permitted under CC BY-NC. No commercial re-use. See rights and permissions. Published by BMJ.

For numbered affiliations see end of article.

Correspondence to

Dr Aitor Martín-Pintado-Zugasti; martinpintado.a@gmail.com

\section{ABSTRACT}

Introduction Neck pain is a very common

musculoskeletal disorder associated with high

socioeconomic costs derived from work absenteeism and medical expenses. Previous studies have suggested that patients with neck pain of different origins present sensorimotor control impairments compared with the asymptomatic population. However, there is a small number of published studies focusing on these with conflicting results. In addition, the existing methodological limitations highlight the need for more and better quality studies. Moreover, longitudinal studies are necessary to investigate whether changes in pain or disability in individuals with chronic neck pain over time associate with changes in cervical sensorimotor control.

Methods and analysis This is a descriptive, observational, longitudinal, prospective study consecutively enrolling 52 patients with non-specific neck pain and 52 age-matched asymptomatic participants.

Intensity of pain, neck disability, duration of symptoms, topography of pain and comorbidities will be registered at baseline. Sensorimotor control variables including active range of motion, movement speed, acceleration, smoothness of motion, head repositioning accuracy and motion coupling patterns will be recorded as primary outcomes by means of inertial sensors during the following tests consecutively performed in two sessions separated by 12 months: (1) kinematics of planar movements, (2) kinematics of the craniocervical flexion movement, (3) kinematics during functional tasks and (4) kinematics of task-oriented neck movements in response to visual targets.

Secondary outcomes will include: (1) Regular physical activity levels, (2) Kinesiophobia, (3) Symptoms related to central sensitisation and (4) The usability of the inertial measurement unit sensor technology.

Ethics and dissemination This study was approved by the Research Ethics Committee of CEU San Pablo University (495/21/39). Patients will be recruited after providing written informed consent and they will be able to withdraw their consent at any time. Only the study

\section{Strengths and limitations of this study}

- The use of technology such as inertial sensors is increasing in research and clinical practice when assessing sensorimotor control, since it allows for the precise electronic record of the data and for feedback options for patients or examiners.

- Prospective study with 1-year follow-up, which allows the investigation of possible alterations in sensorimotor control in patients with neck pain and their influence in the evolution of symptoms.

- The set of multiple variables analysed in the study allow for the evaluation of different skills of sensorimotor control and possible secondary influencing variables such as psychological factors.

- Limitations include potential bias due to selfreported data from questionnaires or the limited sample recruitment of population from one country.

investigators will have access to the study data. The results will be disseminated through scientific publications, conferences and media.

Trial registration number NCT05032911.

\section{INTRODUCTION}

Neck pain is a very common musculoskeletal disorder, with a prevalence in the general population that ranges between $15 \%$ and $50 \%$ worldwide. ${ }^{1}$ In turn, it has been observed that up to $71 \%$ of the adult population has neck pain at some point in their life and is considered the fourth cause of disability worldwide. All this implies an important public health problem associated with high socioeconomic costs derived from work absenteeism and medical expenses. ${ }^{2}$

Previous studies have observed that patients with craniocervical pain of different origins 
present sensorimotor control impairments, ${ }^{3-9}$ which refer to all sensory and motor processes that control muscles and spinal alignment and movement to meet demands of healthy function and loading of the spine. ${ }^{10}$ In this way, the recent existing systematic reviews ${ }^{11-14}$ have suggested that patients with neck pain show alterations in sensorimotor control when compared with the asymptomatic population, including reduced active range of motion, alterations in precision of movement, impaired repositioning accuracy, reduced movement speed or decreased smoothness of neck movements. However, these reviews have highlighted the small number of published studies and the existing methodological limitations and argued that there is a need for a larger number of quality studies evaluating these parameters. Moreover, it has been reported that there is a scarcity of studies that examine cervical spine kinematics during the execution of common functional activities of daily living. ${ }^{11}$

Clinical evaluation of sensorimotor control is believed to offer useful information to understand the underlying mechanisms of neck pain conditions. ${ }^{7}$ In addition, sensorimotor control tests have the potential to be used as a subgrouping tool for patients as well as they could guide intervention strategies for patients with neck pain. ${ }^{13}$ However, it remains unclear whether these sensorimotor control impairments increase the risk of a subsequent neck pain episode or whether they can provide a quantitative method for evaluating the effectiveness of treatment. $^{11}$

Opposite to previous findings, recent research did not observe differences in cervical sensorimotor control outcomes between individuals with non-specific neck pain and asymptomatic individuals ${ }^{15-17}$ and have questioned the clinical usefulness of some sensorimotor control tests for individuals with chronic idiopathic neck pain. ${ }^{17}$ Moreover, a recent longitudinal study ${ }^{16}$ showed that changes in pain or disability in individuals with chronic idiopathic neck pain over 6 months of follow-up did not associate with changes in cervical sensorimotor control, also questioning the clinical relevance of sensorimotor control tests in order to evaluate sensorimotor skills or to guide clinicians' practice. To the authors' knowledge, there is a lack of research which have investigated possible associations between changes in pain or disability, and changes in sensorimotor control over a long-term follow-up of 1 year or above in chronic neck pain patients. Longer follow-up periods might have higher potential to detect these possible relationships, since several patients with chronic neck pain conditions will need for long periods to consistently perceive changes on their clinical evolution.

Meisingset et $a l^{18}$ suggested that factors other than sensorimotor control may explain a larger proportion of the changes in neck pain and disability in patients with non-specific neck pain.

Therefore, there is a need of further research on the possible differences between patients with neck pain and asymptomatic individuals on sensorimotor control and its association with changes on clinical outcomes of pain or disability over time. New technologies, such as wearable sensors, may provide new possibilities for researching the underlying factors involved in sensorimotor control impairments and their influence on activities of daily living. ${ }^{13}$

Our hypothesis is that kinematics will show different characteristics when comparing chronic neck pain patients and asymptomatic individuals in terms of reduced sensorimotor control in patients with chronic neck pain.

The main objective of this study is to compare the sensorimotor control between subjects with neck pain and asymptomatic participants during planar movements, functional tasks, and task-oriented neck movements, in terms of active range of motion, movement speed, acceleration, smoothness and head repositioning accuracy.

Second, we aim to evaluate whether changes in pain or disability associate with changes in the sensorimotor control variables after 1-year follow-up.

Third, we aim to analyse the influence of psychological variables related to pain, such as fear of movement or fear avoidance beliefs, on sensorimotor control outcomes.

\section{METHODS AND ANALYSIS \\ Design}

This is a descriptive, observational, longitudinal, prospective study in patients with neck pain and a control group of asymptomatic participants. The research will include two independent measuring sessions separated by 12 months, when all outcomes of interest (see below) will be recorded.

This study has been designed and the findings will be reported in accordance with the Strengthening the Reporting of Observational Studies in Epidemiology. ${ }^{19}$ The research has been preregistered at www.clinicaltrials. gov (NCT05032911).

\section{Sample and selection}

The sample will be composed of a group of patients with neck pain and another group of asymptomatic subjects. Participants will be enrolled through flyers, distribution of online forms through social networks and email, or direct verbal communication at CEU San Pablo University, the CEU San Pablo University clinic, as well as in private physiotherapy clinics.

Subjects will be eligible to be included in the study if they are between 18 and 65 years old and fulfil the following selection criteria. Patients should have neck pain as their main complaint with an intensity of pain of at least 3 points out of 10 on a Visual Analogue Scale and a neck pain duration of at least 3 months of evolution. Neck pain could be from nonspecific mechanical origin, associated with whiplash or with a previous medical diagnosis of degenerative or inflammatory alterations of the cervical spine, associated or not with headache and pain in the shoulder region or the upper limb or diagnosed with cervical radiculopathy. Patients with neck pain will be excluded if they present any of the following criteria: (1) 
Visual impairment not corrected using glasses/contact lenses, (2) Complex regional syndrome, (3) Previous surgeries in the neck and/or head region, (4) Vertigo/ dizziness, (5) Presence of tumours in the craniocervical region, (6) Previous fracture in the head or neck region and (7) Osseous deformities in the thoracic, cervical or cranial region.

Asymptomatic subjects should not present any pain in the cervical region during the last year and no previous treatment for neck pain in order to be included in the study. The exclusion criteria will be the same described for patients with neck pain.

Once deemed eligible, subjects will be asked to read and sign the informed consent prior to participation and then will be invited to participate in the study by attending to two independent measuring sessions separated by 12 months.

\section{Instrumentation and measures}

Prior to testing, subjects' demographic characteristics such as age, gender, weight, height and level of physical activity will be recorded at the beginning of the first measuring session. In addition, the following descriptive variables will be registered for the group of patients with neck pain: (1) Current intensity of pain (Visual Analogue Scale), (2) Intensity of pain during the last week (Visual Analogue Scale), (3) Intensity of pain during the last month (Visual Analogue Scale), (4) Neck disability measured through the Spanish version of the Neck Disability Index, ${ }^{20}(5)$ Duration of pain (months), (6) Topography of pain (Hand-drawn pain map), (7) Presence of pain in other body regions (lumbar, shoulder, head, etc), (8) medication use and (9) Previous medical diagnosis associated with pain (whiplash, radiculopathies, arthropathies).

In addition, participants will complete questionnaires related to fear of movement, fear avoidance beliefs and central sensitisation. This will be described in detail below.

\section{Primary outcomes: sensorimotor control assessment}

Sensorimotor control variables will be recorded by means of small $(4 \mathrm{~cm} \times 4 \mathrm{~cm} \times 8 \mathrm{~cm})$, light $(<200 \mathrm{~g})$ inertial measurement unit (IMU) sensors (Werium Solutions, Madrid, Spain), which integrate a three-dimensional accelerometer, a gyroscope and a magnetometer. This inertial sensor technology has previously shown good or excellent intrarater and inter-rater reliability in the measurement of cervical range of motion. ${ }^{21} 22$

Two of these wireless wearable sensors will be placed on the forehead (adhered to the centre of the forehead of the subject, defined as the place where the lines that bisect the forehead longitudinally and horizontally cross) and on the T4 spinous process (figure 1). Placement of sensors with these landmarks has been shown to be a reliable method for measuring cervical range of motion in previous research. ${ }^{21}$

An independent assessor will administer all testing procedures, being blinded to subjects' group allocation (Neck pain or asymptomatic). As it has been reported

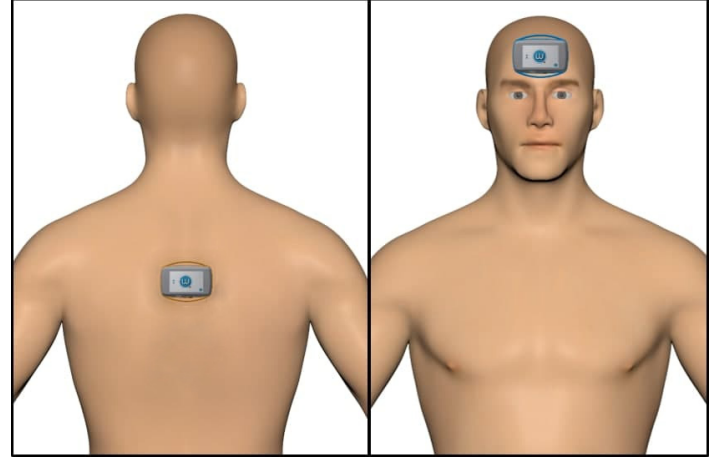

Figure 1 Placement of wearable inertial sensors on the forehead and T4.

by similar previous research, ${ }^{16}{ }^{23}$ bias during this type of testing procedures is unlikely, since these procedures use automatic computerised data collection.

The following tests will be randomly performed by each subject enrolled in the study in two measuring sessions separated by 12 months: (1) kinematics of planar movements (Planar directions sequence will be also randomised), (2) kinematics of the craniocervical flexion movement, (3) kinematics during functional tasks and (4) kinematics of task-oriented neck movements in response to visual targets. All sensorimotor control variables analysed for each test are described below.

During the test, the assessor will be able to monitor on real time the range of motion values displayed on a computer screen (figure 2).

The usability of the IMU sensor technology will be evaluated through the Spanish version of the System Usability Scale (SUS) ${ }^{24}$ It includes 10 statements about the perceived usability of the game on a scale from 0 to 4 . To obtain the final SUS score, the sum of the patients' answers is multiplied by 2.5 . Therefore, the final score ranges from 0 (low usability) to 100 (high usability).

\section{Kinematics of planar movements}

We will measure kinematics of planar movements in the three anatomical planes, as described in a similar way to this study in previous research. ${ }^{8}$ Subjects will be asked to perform three consecutive active movements in each plane in a sitting posture. The sequence of the movement planar directions (flexion-extension, right-left rotation and right-left lateral flexion) will be randomised using a computer software (Research Randomiser: https://www. randomizer.org/). This procedure will be performed by each subject in three different ways in a consecutive order: (1) open eyes at subject's own pace, (2) closed eyes at subject's own pace and (3) open eyes at maximal velocity (Movements at subject's own pace can serve as a warm-up before the more intense maximal velocity movement).

A set of verbal and visual standardised instructions will be prerecorded and displayed on a computer in order to provide identical detailed instructions for all subjects (see online supplemental appendix 1). 


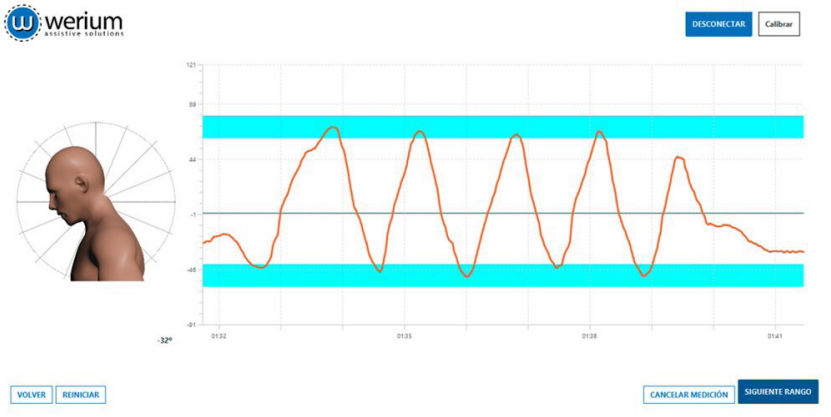

Figure 2 Example of real-time range of motion values displayed on a computer screen.

Initially, study subjects will be asked to sit naturally in a standard chair with the feet well supported on the floor and the neck and head in a neutral comfortable position with their hands resting on their thighs. Then, the recorded standardised instructions will be played on the computer to explain the procedure. The assessor will ask the subjects whether they understood all the measuring protocol or had any question about it before starting. Then, the sensors will be placed on the forehead and T4 spinous process and the subjects will be reminded to stay and memorise the starting neutral position of the head and reproduce this position as accurately as possible after the repetitions of each movement. At this moment, sensors will be calibrated, and the subject will be asked to perform the three repetitions of the first randomised planar movement. Sensors will be again calibrated at the starting position before the start of the motion in all remaining planar movements. Participants will perform a total of nine repetitions (three flexion-extension, three right-left rotation and three right-left lateral flexion) for each of three types of movement: (1) open eyes at subject's own pace, (2) closed eyes at subject's own pace and (3) open eyes at maximal velocity.

The protocol will be interrupted in case the patient reports worsening of the symptoms, dizziness and/ or vertigo which limit their capacity to perform the measuring tests. In case a patient reports any mild tolerable secondary effect which did not limit their performance of the test, data on which test was associated with secondary effects and the characteristics of the effect will be recorded. Patients whose measurement had to be interrupted will be offered to participate again on a different date.

\section{Kinematics of the craniocervical flexion movement}

After the planar movements' protocol, subjects will be asked to perform three consecutive active movements of the craniocervical flexion movement in two different positions (Consecutively performed to facilitate learning how to perform the movement in supine position first): (1) supine and (2) sitting. A set of verbal and visual standardised instructions will be prerecorded and displayed on a computer in order to provide identical detailed instructions for all subjects (see online supplemental appendix 1). In addition, the movement will be demonstrated to the participant, so they can also directly observe its execution.

For the movement in supine position, only the sensor of the forehead will be used, since the T4 sensor would be compressed against the table. Participants will be placed in a relaxed supine position with the forearms resting on the abdomen, the knees flexed and the neck in a neutral position with the face and the line bisecting the neck longitudinally being horizontal to the plinth. Subjects will be reminded to stay and memorise the starting neutral position of the head and reproduce this position as accurately as possible after each of the three repetitions. At this moment, sensors will be calibrated and the subject will be asked to perform three repetitions of the craniocervical flexion movement as far as possible, consisting on an anterior rotation of the head in a nod action, feeling the back of their heads slide up on the table. During the test, the assessor will provide verbal cues to guide the process with a correct technique if necessary.

Additionally, the measure will be repeated in case the assessor detects signs of compensation, such as lower cervical flexion or neck retraction.

A similar protocol will be used for the craniocervical flexion movement in sitting position. Subjects will be asked to sit naturally in a standard chair with the feet well supported on the floor and the neck and head in a neutral comfortable position with their hands resting on their thighs. In this case, patients will be instructed to perform three repetitions of the craniocervical flexion movement as far as possible, consisting on an anterior rotation of the head in a nod action, avoiding any lower flexion movement, so the head occiput remains in the same location.

\section{Kinematics during functional tasks}

Then, subjects will be asked to perform two different functional movements on their own pace (sequence will be randomised): (1) Upper limb functional task and (2) drinking water task. A set of verbal and visual standardised instructions will be prerecorded and displayed on a computer in order to provide identical detailed instructions for all subjects (see online supplemental appendix 1).

The upper limb functional task will be based on the protocol previously designed by Tsang et $a l^{7}{ }^{7}$ Subjects will be asked to sit naturally in a standard chair with the feet well supported on the floor and the neck and head in a neutral comfortable position. Then, they will be asked to transfer a $2 \mathrm{~kg}$ weight (placed on their thigh) and lift and release it on an elevated platform (located $70 \mathrm{~cm}$ above the subject's thigh, $30 \mathrm{~cm}$ in front of the patient's knee and $30 \mathrm{~cm}$ lateral to the patient's acromion) and finally pick it again to transfer back to the original location on the thigh. This process will be repeated three times after the sensors are calibrated in the neutral starting position.

The drinking water task will start with the patient sitting in the same starting position. After calibrating the sensor 


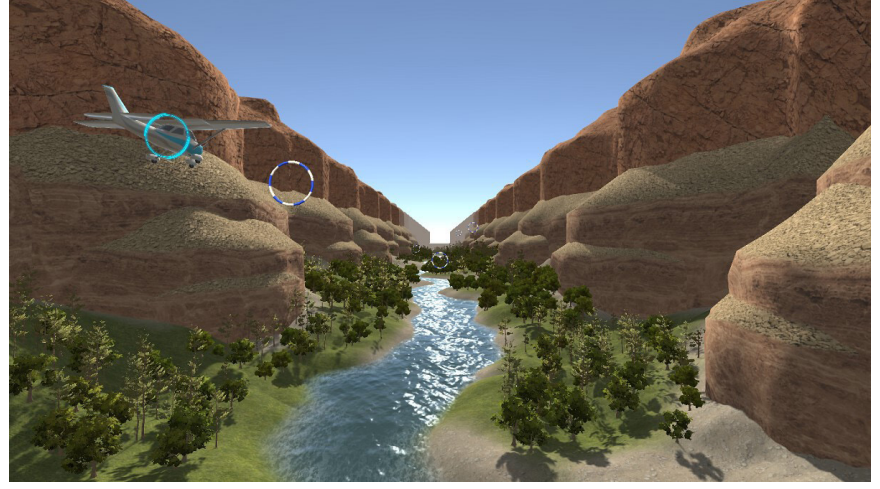

Figure 3 Task-oriented virtual reality game.

in the starting neutral position, patients will be asked to pick a glass of water (placed on a table $30 \mathrm{~cm}$ above and $30 \mathrm{~cm}$ in front of the patients' knee), drink a sip of water and transfer the glass back to its initial placement. This process will be repeated three times, asking the patient to return to the starting position between each repetition.

\section{Kinematics of task-oriented neck movements in response to visual targets}

Participants will first receive standardised instructions about the activity, consisting of a serious game in a virtual environment (Active Airlines) designed for the assessment and exercise of the cervical spine. This is a Windows-based application (using the C\# language in Unity IDE) designed to provide an interactive scenario that allows the assessment of multiple motor control parameters. The main functionality of the software solution was to control a virtual aeroplane to reach targets using the head motion (figure 3). Motion of the head and neck controls the movement of an aeroplane to target different reference points (randomly located) to get a specific score (1 point per target). Previous studies have described the use of similar virtual reality games to assess sensorimotor control, in which an aeroplane must reach different goals through head and neck. ${ }^{25}$

Subjects will be again asked to sit naturally in a standard chair with the feet well supported on the floor and the neck and head in a neutral comfortable position with their hands resting on their thighs. Then, the main features of the game will be explained, and the participant will first play the game as a warmup to also understand its functioning (Game finishes when the subject reaches a total of 15 targets during approximately $1 \mathrm{~min}$ ). Then, the same process will be repeated as the real trial for data collection.

\section{Sensorimotor control data processing}

The software application computes the complete range of motion of the participants, expressed as angles from the calibrating starting position in the three axes, sampled every $20 \mathrm{~ms}$ from each sensor (forehead and T4). This information will be exported in .csv using RStudio (library 'readr'). In order to obtain the sensorimotor control variables, a data frame (data.frame() function) will be created thanks to an iterative process which should read each one of the exported .csv files and extract the corresponding data. Each row will correspond to one subject, and the columns will represent all the sensorimotor control variables that can be obtained from the raw data, described below. In addition, data smoothing filters will be applied to avoid peaks and variations not corresponding to the trend of each data set.

The analysis of these data will allow for the calculation of the following variables for all the sensorimotor control tests described above:

- Active range of motion expressed as the maximal angular displacement $\left({ }^{\circ}\right)$ : characterised using two different perspectives: as three full-movements (flexion-extension, lateral flexion and rotation) or as six half movements (flexion, extension, right lateral flexion, left lateral flexion, right rotation and left rotation).

- Movement speed expressed as angular velocity $(\% / \mathrm{s})$ : calculated as the discrete derivative of angular orientation applying a standard smooth filtering algorithm. Then, the mean and the peak velocity are calculated for each of the six half movements.

- Acceleration $\left(\% / \mathrm{s}^{2}\right)$ : calculated as the second discrete derivate of the angular orientation and expressed as mean or peak acceleration for each of the six half movements.

- Smoothness of motion expressed as movement jerk $\left({ }^{\circ} / \mathrm{s}^{3}\right)$ : calculated as the third discrete derivative of the angular orientation (Change in acceleration) for each of the six half movements. ${ }^{415}$

- Head repositioning accuracy expressed as angular displacement $\left({ }^{\circ}\right)$ : calculated as the repositioning error considering the difference between the neutral starting position and the following neutral positions reached after the performance of any of the movements described.

- Motion coupling patterns $\left({ }^{\circ}\right)$ : calculated as the angular displacement occurring in a different anatomical plane to the one that is being tested for each of the planar movements.

\section{Secondary outcomes}

We will record the following secondary outcomes (Auto-administered questionnaires) at the end of each measuring session. In case of the questionnaires related to pain, healthy participants without neck pain will fulfil them by considering their perception in relation to previous experiences of pain.

Regular physical activity will be measured using the Spanish version of the short version of the International Physical Activity Questionnaire. ${ }^{26}$ It evaluates activity in the last 7 days and classifies it into three types (walk, moderate physical activity and intense physical activity) and by estimated energy expenditure for each of them (3.3, 4.0 and 8.0 Metabolic Equivalents of Task (METs)). Based on this data, participants are stratified by three activity levels (low, intermediate and high) and METs-min/week can be calculated. 
The Spanish version of the Tampa Scale for Kinesiophobia- $11^{27}$ will be used to assess fear of movement and injury. It is rated on a 4-point scale, where a 4 represents 'strongly agree' with the statement and a 1 represents a 'strongly disagree'. Scores range from 11 to 44 with higher scores indicating higher fear of movement.

Pain-related fear-avoidance will be measured using the Spanish version of the Fear Avoidance Components Scale (FACS) ${ }^{28}$ The FACS includes 20 items, scored on a 6-point Likert scale, for a total score range from 0 to 100. Total scores indicate one of the following severity levels: subclinical (0-20); mild (21-40); moderate (41-60); severe (61-80) and extreme (81-100).

Symptoms related to central sensitisation will be measured with the Spanish version of the Central Sensitisation Inventory. ${ }^{29}$ It has two parts; part A assesses 25 health-related symptoms common to central sensitisation (items scored from 0 to 4 ), with a total score ranging from 0 to 100, and part B (which is not scored) asks about previously diagnosis of one or more specific disorders.

\section{Study procedure}

The study duration per participant will be of 1 year. The timeline of the study procedure from a participant's perspective will include all aspects described in the method section in the following order: participant enrolment in the study, evaluation of selection criteria for eligibility and invitation to attend to the first measuring session once deemed eligible. This session includes the following sequence of measurements: initial record of demographic variables, registration of descriptive variables related to pain, record of primary outcomes (Sensorimotor control assessment) in a random order, including kinematics of planar movements, kinematics of the craniocervical flexion movement, kinematics during functional tasks and kinematics of task-oriented neck movements in response to visual targets. Finally, patients will fulfil the set of autoadministered questionnaires.

All measurements included in the first session will be repeated in the same sequence in the second measuring session 1 year after the date of the first session.

The study procedure is expected to start in January 2022 and data collection is expected to conclude in the second quarter of 2023.

\section{Data analysis plan}

All data will be analysed using the Statistical Package for Social Sciences (SPSS) software V.24.0 (SPSS) and R software. A normal distribution of quantitative data will be assessed by the Kolmogorov-Smirnov test and the mean and SD for each normally distributed quantitative variable will be analysed.

Multiple Independent sample t-test or Mann-Whitney $\mathrm{U}$ test will be conducted to compare baseline age, weight, height, body mass index (BMI) and level of physical activity between groups between the two groups when corresponds.
In order to determine whether there are differences in sensorimotor variables across times, and also to determine whether other variables such as pain, disability, and fear of movement or fear avoidance beliefs influence sensorimotor outcomes, a two-stage analysis will be conducted. First, a simple linear regression will be done to analyse the relation between each dependent variable (ie, sensorimotor variables) with each of the independent variables (eg, age, weight, height, BMI and level of physical activity, pain, disability, fear avoidance, kinesiophobia and central sensitisation). Separate analyses will be conducted for each outcome. Significant variables in the univariate analysis at $p \leq 0.20$ will be added to a linear mixed effect model. This $p$ value has been suggested by some as a conservative criterion to involve all potential variables that could be significant in a multivariable regression model. More traditional alpha levels can fail in identifying variables that could be important. We will examine for multicollinearity among the independent variable(s) using the variance-inflation facto. In addition, we will check the linearity assumption by inspecting the residuals. Model building will be performed using forward stepwise regression analysis and competing models will be compared using the Akaike information criterion and the Bayesian information criteria.

Outliers, those who do not perform the test correctly or exposed to technical problems will be dropped from the analyses. For all analyses, statistical significance will be set at $\mathrm{p}<0.05$.

\section{Sample size}

The sample size of this study was determined using G*Power, V.3.1.9.2 (Franz Faul, University at Kiel, Germany), considering the results from a pilot study with 20 subjects: 10 asymptomatic and 10 neck pain subjects. ${ }^{30}$ A sample size calculation was conducted for the variables described above during the execution of each of the tests performed. The variable with the largest sample size estimate was the peak velocity in planar movement rotation with closed eyes at subject's own pace.

Sample size was calculated using a two-group, one-tailed t-test with 0.95 power (1-beta error probability), an alpha level of 0.5 and power of 0.8 . A sample size of 104 individuals (52 per group) was estimated considering an effect size of 0.65 , a velocity score $(\% / \mathrm{s}) \pm \mathrm{SD}$ in the control group of $151.01 \pm 49.39$ and of $123.87 \pm 31.80$ in the neck pain group.

\section{DISCUSSION}

The objectives of this observational study protocol aim to provide further evidence on the analysis of chronic neck pain and its possible related mechanisms regarding sensorimotor control and other variables, such as psychological factors related to pain.

Recent systematic reviews have highlighted the need of further quality research, including larger sample sizes of patients with chronic neck pain in order to investigate 
sensorimotor control during functional movements, ${ }^{11}$ analytical movements, static postures ${ }^{13}$ or specifically designed tests. $^{12}$

Sensorimotor control describes the sensory, motor and central integration and processing components involved in maintaining joint homeostasis during bodily movements. ${ }^{31}{ }^{32}$ Multiple subsystems are addressed when testing sensorimotor control, including proprioceptive sensory inputs, kinesthesia, vestibular or oculomotor control, so they can be globally addressed or aiming to isolate the subsystem(s) of interest. ${ }^{12}$

This study protocol includes a set of multiple procedures in order to address most of aspects related to sensorimotor control and differentiate some of the subsystems related to it. For instance, the analysis of kinematics of planar movements with closed eyes aims to remove the sensory input from the oculomotor subsystem, while the kinematics of open eyes at maximal velocity may be specially influenced by psychological factors of kinesiophobia. The kinematic analysis of craniocervical flexion isolates the motor control output of deep cervical flexor muscles, which are believed to play an important role in the neck pain mechanisms, due to their capacity to control segmental alignment of the cervical spine. ${ }^{33}$ Furthermore, the analysis of kinetics during functional tasks addresses sensorimotor control in general, as it provides an assessment of a patient's ability to complete activities of daily living that affect their quality of life. ${ }^{11}$

Our hypothesis is that kinematics of most of these movements in patients with chronic neck pain will show impaired sensorimotor control when compared with asymptomatic individuals. However, how these differences may be influenced by other factors (eg, age, pain intensity, disability, fear avoidance beliefs or kinesiophobia) when performing regression analysis of the data remains unclear to us.

The results of this study may have implications for clinical practice, since they can provide useful information to understand the underlying mechanisms of neck pain through the use of new technologies to objectively evaluate sensorimotor control. The sensorimotor control characteristics of patients could potentially be used as a subgrouping tool to guide more targeted interventions. Moreover, the results regarding possible associations between clinical outcomes and sensorimotor control over 1 year in patients with neck pain could provide useful information about the utility of sensorimotor control analysis in clinical practice, considering that recent research have questioned the clinical relevance of some specific cervical sensorimotor control tests. ${ }^{16}$

This study protocol has some limitations. The study population characteristics may be limited to individuals of European descent. Also, although the instructions to perform each test were standardised, subjects of older age or with lower educational level may present any difficulties to understand the performance of each movement.

Strengths of the study include the large sample of both neck pain and asymptomatic participants, the inclusion of a set of various sensorimotor control tests capable of assessing global and specific aspects of sensorimotor control, the inclusion of multiple variables to describe patients' characteristics (eg, demographics, pain or psychological factors), the long follow-up period and the use of inertial sensor technology that allows for the precise electronic record of the data with feedback options for the examiners.

\section{Patient and public involvement}

Participants were involved in the training and design of the protocol described above. Their perceptions regarding the execution of each test and the ease to perform the study protocol were asked and considered when planning the method of this project.

After finishing the study, a subgroup of participants will be interviewed regarding their experiences of participating in the study and completing all test procedures. In addition, a summary of the results of the study will be disseminated to all study participants via email. In addition, since some population included in the study will be recruited from a university community, all participants will be invited to attend to any presentation about the study results across the university.

\section{Ethics and dissemination}

The study was approved by the Research Ethics Committee of CEU San Pablo University (495/21/39).

Participants will provide informed written consent before being enrolled into the study and they will be able to withdraw their consent at any time during the study, in compliance with the WHO standards and the Declaration of Helsinki. ${ }^{34}$ Only the study investigators will have access to the study data. After the study is completed, the files containing the private information will be destroyed and participants will not be identified through any data, transcripts or publications.

We aim to disseminate the findings of our research through national or international scientific meetings or conferences and in articles published in peer-reviewed scientific journals. This project may also be part of a $\mathrm{PhD}$ thesis. All participants in the study who request it will be sent a summary of the results of the study or the published articles.

\section{Author affiliations}

${ }^{1}$ Departamento de Fisioterapia, Facultad de Medicina, CEU Universities, Universidad San Pablo CEU, Madrid, Spain

${ }^{2}$ Faculty of Business and Social Sciences, University of Applied Sciences, Osnabrück, Germany

${ }^{3}$ Department of Physical Therapy, Faculty of Rehabilitation Medicine, 3-48 Corbett Hall, University of Alberta, Edmonton, Alberta, Canada

${ }^{4}$ Department of Physical Therapy, Occupational Therapy, Rehabilitation and Physical Medicine, Rey Juan Carlos University, Madrid, Spain

${ }^{5}$ La Paz Hospital Institute for Health Research, IdiPAZ, Madrid, Spain

${ }^{6}$ Grupo Multidisciplinar de Investigación y Tratamiento del Dolor, Grupo de

Excelencia Investigadora URJC-Banco de Santander, Madrid, Spain

${ }^{7}$ Departamento de Tecnologías de la Información, Escuela Politécnica Superior, CEU Universities, Universidad CEU San Pablo, Madrid, Spain

${ }^{8}$ Werium Solutions, Arganda del Rey, Madrid, Spain

Contributors TP-F, SL, FM, JF-C, AM-P-Z and EBC: conception and design of the study; FM, PC, EBC and OR-L: recruitment and testing for pilot analysis; EBC, 
TP-F, SL, SA-0, OR-L and AM-P-Z: writing/editing of final manuscript; JF-C and SA-0: critical review and revision of manuscript; RR, CS, SA-0: technical support and statistical analyses. All authors reviewed and approved the manuscript before submission.

Funding The study was supported by the Agencia Estatal de Investigación, Spanish Ministry of Science, Innovation and Universities (Reference number: PID2019-108616RA-I00/AEI/10.13039/501100011033)

Competing interests RR is the CEO of Werium Solutions, manufacturer of the inertial wearable sensor used in the study. The other authors declare no conflict of interest.

Patient consent for publication Not applicable.

Provenance and peer review Not commissioned; externally peer reviewed.

Supplemental material This content has been supplied by the author(s). It has not been vetted by BMJ Publishing Group Limited (BMJ) and may not have been peer-reviewed. Any opinions or recommendations discussed are solely those of the author(s) and are not endorsed by BMJ. BMJ disclaims all liability and responsibility arising from any reliance placed on the content. Where the content includes any translated material, BMJ does not warrant the accuracy and reliability of the translations (including but not limited to local regulations, clinical guidelines, terminology, drug names and drug dosages), and is not responsible for any error and/or omissions arising from translation and adaptation or otherwise.

Open access This is an open access article distributed in accordance with the Creative Commons Attribution Non Commercial (CC BY-NC 4.0) license, which permits others to distribute, remix, adapt, build upon this work non-commercially, and license their derivative works on different terms, provided the original work is properly cited, appropriate credit is given, any changes made indicated, and the use is non-commercial. See: http://creativecommons.org/licenses/by-nc/4.0/.

\section{ORCID iDs}

Filippo Moggioli http://orcid.org/0000-0002-1100-0466

Tomas Pérez-Fernández http://orcid.org/0000-0001-9640-1324

Aitor Martín-Pintado-Zugasti http://orcid.org/0000-0003-3945-8222

\section{REFERENCES}

1 Cohen SP. Epidemiology, diagnosis, and treatment of neck pain. Mayo Clin Proc 2015;90:284-99.

2 Hoy D, March L, Woolf A, et al. The global burden of neck pain: estimates from the global burden of disease 2010 study. Ann Rheum Dis 2014;73:1309-15.

3 Meisingset I, Woodhouse A, Stensdotter AK. Evidence for a general stiffening motor control pattern in neck pain: a cross sectional study pathophysiology of musculoskeletal disorders. BMC Musculoskeletal Disorders 2015;16.

4 Sjölander P, Michaelson P, Jaric S, et al. Sensorimotor disturbances in chronic neck pain--range of motion, peak velocity, smoothness of movement, and repositioning acuity. Man Ther 2008;13:122-31.

5 Sarig Bahat H, Weiss PL, Laufer Y. The effect of neck pain on cervical kinematics, as assessed in a virtual environment. Arch Phys Med Rehabil 2010;91:1884-90.

6 Sarig Bahat H, Weiss PLT, Sprecher E, et al. Do neck kinematics correlate with pain intensity, neck disability or with fear of motion? Man Ther 2014;19:252-8.

7 Tsang SMH, Szeto GPY, Lee RYW. Altered spinal kinematics and muscle recruitment pattern of the cervical and thoracic spine in people with chronic neck pain during functional task. J Electromyogr Kinesiol 2014;24:104-13.

8 Tsang SMH, Szeto GPY, Lee RYW. Movement coordination and differential kinematics of the cervical and thoracic spines in people with chronic neck pain. Clin Biomech 2013;28:610-7.

9 Salehi R, Rasouli O, Saadat M, et al. Cervical movement kinematic analysis in patients with chronic neck pain: a comparative study with healthy subjects. Musculoskelet Sci Pract 2021;53:102377.

10 Brumagne S, Diers M, Danneels L, et al. Neuroplasticity of sensorimotor control in low back pain. J Orthop Sports Phys Ther 2019;49:402-14.

11 Moghaddas D, de Zoete RMJ, Edwards S, et al. Differences in the kinematics of the cervical and thoracic spine during functional movement in individuals with or without chronic neck pain: a systematic review. Physiotherapy 2019;105:421-33.

12 de Zoete RMJ, Osmotherly PG, Rivett DA, et al. Sensorimotor control in individuals with idiopathic neck pain and healthy individuals: a systematic review and meta-analysis. Arch Phys Med Rehabil 2017;98:1257-71.

13 Hesby BB, Hartvigsen J, Rasmussen $\mathrm{H}$, et al. Electronic measures of movement impairment, repositioning, and posture in people with and without neck pain-a systematic review. Syst Rev 2019;8:220.

14 Stenneberg MS, Rood M, de Bie R, et al. To what degree does active cervical range of motion differ between patients with neck pain, patients with whiplash, and those without neck pain? A systematic review and meta-analysis. Arch Phys Med Rehabil 2017;98:1407-34.

15 Lemmers GPG, Heijmans MWM, Scafoglieri A, et al. Threedimensional kinematics of the cervical spine using an electromagnetic tracking device. differences between healthy subjects and subjects with non-specific neck pain and the effect of age. Clin Biomech 2018;54:111-7.

16 de Zoete RMJ, Osmotherly PG, Rivett DA, et al. Cervical sensorimotor control does not change over time and is not related to chronic idiopathic neck pain characteristics: a 6-month longitudinal observational study. Phys Ther 2020;100:268-82.

17 de Zoete RMJ, Osmotherly PG, Rivett DA, et al. No differences between individuals with chronic idiopathic neck pain and asymptomatic individuals on 7 cervical sensorimotor control tests: a cross-sectional study. J Orthop Sports Phys Ther 2020;50:33-43.

18 Meisingset I, Stensdotter A-K, Woodhouse A, et al. Neck motion, motor control, pain and disability: a longitudinal study of associations in neck pain patients in physiotherapy treatment. Man Ther 2016;22:94-100.

19 von Elm E, Altman DG, Egger M, et al. The strengthening the reporting of observational studies in epidemiology (STROBE) statement: guidelines for reporting observational studies. Bull World Health Organ 2007;85:867-72.

20 Andrade Ortega JA, Delgado Martínez AD, Almécija Ruiz R. Validation of the Spanish version of the neck disability index. Spine 2010;35:E114-8.

21 Raya R, Garcia-Carmona R, Sanchez C, et al. An inexpensive and easy to use cervical range of motion measurement solution using inertial sensors. Sensors 2018;18:2582-17.

22 Pérez-Fernández T, Armijo-Olivo S, Liébana S, et al. A novel use of inertial sensors to measure the craniocervical flexion range of motion associated to the craniocervical flexion test: an observational study. J Neuroeng Rehabil 2020;17:152.

23 de Zoete RMJ, Osmotherly PG, Rivett DA, et al. No differences between individuals with chronic idiopathic neck pain and asymptomatic individuals on seven cervical sensorimotor control tests: a cross-sectional study. J Orthop Sports Phys Ther 2019;23:1-37.

24 Sevilla-Gonzalez MDR, Moreno Loaeza L, Lazaro-Carrera LS, et al. Spanish version of the system usability scale for the assessment of electronic tools: development and validation. JMIR Hum Factors 2020;7:e21161

25 Sarig H, Chen X, Reznik D. Interactive cervical motion kinematics : Sensitivity, speci fi city and clinically signi fi cant values for identifying kinematic impairments in patients with chronic neck pain. Manual Therapy 2014:1-8.

26 Roman-Viñas B, Serra-Majem L, Hagströmer M, et al. International physical activity questionnaire: reliability and validity in a Spanish population. Eur J Sport Sci 2010;10:297-304.

27 Gómez-Pérez L, López-Martínez AE, Ruiz-Párraga GT. Psychometric properties of the Spanish version of the Tampa scale for Kinesiophobia (TSK). J Pain 2011;12:425-35.

28 Cuesta-Vargas Al, Neblett R, Gatchel RJ, et al. Cross-Cultural adaptation and validity of the Spanish fear-avoidance components scale and clinical implications in primary care. BMC Fam Pract 2020;21:1-9.

29 Cuesta-Vargas Al, Roldan-Jimenez C, Neblett R, et al. Cross-cultural adaptation and validity of the Spanish central sensitization inventory. Springerplus 2016;5:1837.

30 Faul F, Erdfelder E, Lang A-G, et al. G*Power 3: a flexible statistical power analysis program for the social, behavioral, and biomedical sciences. Behav Res Methods 2007;39:175-91.

31 Turvey MT, Fonseca S. Nature of motor control: perspectives and issues. Adv Exp Med Biol 2009;629:405-21.

32 Riemann BL, Lephart SM. The sensorimotor system, part I: the physiologic basis of functional joint stability. J Athl Train 2002;37:71-9.

33 Jull GA, O'Leary SP, Falla DL. Clinical assessment of the deep cervical flexor muscles: the craniocervical flexion test. J Manipulative Physiol Ther 2008;31:525-33.

34 World Medical Association. World Medical association Declaration of Helsinki: ethical principles for medical research involving human subjects. JAMA 2013;310:2191-4. 